\title{
Mast Cell Interaction with Neutrophils in Human Gastric Carcinomas: Ultrastructural Observations
}

\author{
Antonio Ieni, Valeria Barresi, Giovanni Branca, \\ Rosario Alberto Caruso, and Giovanni Tuccari \\ Department of Human Pathology "Gaetano Barresi", University of Messina and \\ Azienda Ospedaliera Universitaria "Policlinico Gaetano Martino", 98125 Messina, Italy
}

Correspondence should be addressed to Antonio Ieni; aieni@unime.it

Received 15 August 2016; Accepted 27 October 2016

Academic Editor: Consuelo Amantini

Copyright (C) 2016 Antonio Ieni et al. This is an open access article distributed under the Creative Commons Attribution License, which permits unrestricted use, distribution, and reproduction in any medium, provided the original work is properly cited.

\begin{abstract}
Aim. The role of mast cells in cell-cell immune interactions has received increasing attention, although their functional interaction with neutrophils still remains to be clarified in tumors. The aim of the present study was to investigate the association between mast cells and neutrophils in a series of gastric carcinomas (GC). Patients and Methods. 52 surgically resected GC specimens were routinely processed for both light and electron microscopy. Only cases showing both mast cells and neutrophils in the tumor stroma were considered in the analysis. Results. Only 9 GC (M:F = 5:4; age range: 50-82 years) showed both mast cells and neutrophils in the tumor stroma. At ultrathin sections, we identified heterotypic aggregation and intermingling of mast cells and neutrophils. Mast cells had mature phenotype and showed full complement of granules with homogeneous, scroll, particle, and mixed pattern. In addition, we found normal-appearing or early apoptosis showing neutrophils. Conclusion. Our histological findings showed the likely interaction between mast cells and neutrophils in GC. We hypothesize that the granular content of mast cells may be released in small quantity through a mechanism called "kiss-and-run fusion," which is alternative to well-known massive anaphylactic or piecemeal degranulation.
\end{abstract}

\section{Background}

Despite its decreasing incidence and mortality, gastric cancer (GC) still represents the second leading cause of cancer death worldwide [1-3]. Approximately, $20 \%$ of cancer deaths worldwide are associated with unresolved infection or chronic inflammation; moreover, prolonged inflammation can lead to mucosal atrophy, which in some patients precedes neoplastic development [4]. In particular, unresolved inflammation may create a microenvironment facilitating cellular transformation and chronic tissue damage. In addition, it may trigger repair response including growth and survival factors, tissueremodeling enzymes, and immune regulatory cytokines [5]. However, the role of immune cell populations in gastric cancerogenesis has not been yet fully clarified [4]. Neutrophils, mast cells, eosinophils, and dendritic cells may directly infiltrate foveolar epithelium, whereas the lamina propria is permeated by mononuclear cells, such as lymphocytes, macrophages, and plasma cells [6-10].
By virtue of their strategic position, mast cells represent a major sensory arm of the innate immune system $[11,12]$. Mast cells are granulated tissue-resident cells of hematopoietic lineage which may be found near epithelium or at the intraepithelial level as well as near the vessels in the connective tissue $[9,11,12]$. Mast cells are a prominent source of proinflammatory mediators and cytokines that can induce inflammation, vascular changes, and leukocyte infiltration $[11,12]$. In particular, mast cell-derived tumor necrosis factoralpha plays an important role in inflammation through the recruitment of neutrophils in the sites of infection [13, 14]. However, mast cell degranulation is also responsible for the characteristic signs of inflammation and neutrophil infiltration around the wound site $[15,16]$. Recently, mast cells have received increasing attention as emerging protagonists in cell-cell immune interaction [17-20]. Although functional interaction between mast cells and neutrophils has not been yet clarified, morphological heterotypic aggregation between these two cell populations has been described in 


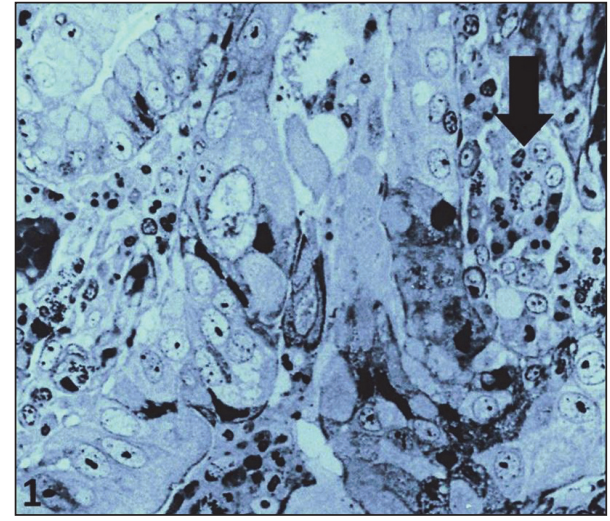

FIGURE 1: Mast cell surrounded by neutrophils (arrow) in the tumor stroma of gastric adenocarcinoma. (semithin section, $\times 200$ ).

mouse experimental studies on contact dermatitis [21]. In the present study, we report morphological interaction between mast cells and neutrophils in the stroma of human GC.

\section{Materials and Methods}

52 surgically resected GC specimens were initially considered. None of the patients had undergone preoperative irradiation or immunochemotherapy. All samples were routinely processed for both light and electron microscopy. Briefly, fresh tumor specimens were divided into two portions by using a sharp razor blade. One portion was $10 \%$ formalinfixed, paraffin-embedded, and stained with hematoxylin and eosin (H\&E). The other portion was minced into smaller pieces and immediately fixed in 3\% phosphate-buffered glutaraldehyde, $\mathrm{pH} 7.4$, and postfixed in 1\% osmium tetroxide for electron microscopy. Semithin Giemsa-stained sections were reviewed by light microscopy to select cases showing both mast cells and neutrophils in the tumor stroma. Tissue blocks of selected cases were cut by using Ultratome III, LKB (Stockholm-Bromma, Sweden), to obtain ultrathin sections, which were double-stained with uranyl acetate and lead citrate and finally examined and photographed under a JEOL 1200 EX TEM electron microscope (JOEL, Tokyo, Japan) at $70 \mathrm{kV}$.

\section{Results}

Of the 52 GC specimens initially considered, only 9 showed both mast cells and neutrophils in the tumor stroma. Those cases included five males and four females (age ranging between 50 and 82 years). Tumor diameter ranged between two and eight $\mathrm{cm}$. According to Laurèn classification, six cases were intestinal-type GC and three were diffuse-type GC [22]. All cases were at stage IIA or IIB; $6 / 9$ were with N0 status, while three were T3N1/T2N2.

In Giemsa-stained semithin sections, mast cells were easily identified by the presence of typical granules (Figure 1, arrow). Generally, they occurred as single cells or occasionally in clusters containing one to two mast cells and one to four neutrophils (Figure 1).
Ultrathin sections revealed the presence of heterotypic aggregation and intermingling of mast cells and neutrophils (Figures 2(a) and 2(b)). In detail, mast cells had mature phenotype with full complement of granules showing homogeneous, scroll, particle, and mixed pattern (Figures 2(a) and 2(b)). In other areas, mast cells showed peripheral location of granules (Figures 2(b) and 2(c)) and sometimes granules were localized close to the cell membrane (Figures 2(c) and 2(d)). We also found few swollen granules and numerous cytoplasmic microtubules (Figures 2(c) and 2(d)) and association of microtubules and granules (Figure 2(c)). Neutrophils located close to mast cells were morphologically well preserved or exhibited ultrastructural features of early apoptosis, such as nuclear chromatin separation into dense and electron-lucent areas, preservation of cytoplasmic granules, focal loss of glycogen particles, and preserved plasma membrane (Figures 2(a), 2(b), 2(c), and 2(d)).

\section{Discussion}

Previous experimental studies have ultrastructurally documented close spatial relationship between mast cells and neutrophils in IgE-mediated late-phase cutaneous response of mouse contact dermatitis [21]. In detail, mast cells contained nonfused swollen granules filled with altered contents and showed extrusion of membrane-free granules through membrane pores [21]; interestingly, few mast cells secreted membrane-bound granules into the dermis without cell membrane damage [21]. Successively, the number of mast cells prominently increased and total serum IgE level was greatly elevated; in addition, mast cells which showed typical anaphylactic degranulation and several immature mast cells, characterized by well-developed Golgi apparatus, were also observed [21]. To our knowledge, the above-mentioned ultrastructural features of mast cell-neutrophil interaction have not been described either in human or in experimental neoplasms thus far. Therefore, this study is the first to document the morphological relationship between mast cells and neutrophils in human GC. Nonetheless, we are not able to explain why this close cellular association occurred in only 9 of 52 GC independently from gender and age of the patients and histotype of the tumor. Nevertheless, the evidence that all cases were stage IIA/IIB may suggest the appearance of abovementioned cellular relationship in low stage, less aggressive GC.

The morphological ultrastructural features which we observed in this series of GC can be summarized as follows: (1) whenever present, mast cells had large areas of close contact with neutrophils; (2) several neutrophils exhibited satellitosis-like pattern around mast cells; (3) mast cells close to neutrophils were functionally activated as documented by the presence of numerous cytoplasmic microtubules and fewer granules peripherally located; (4) neutrophils were well preserved or showed early apoptotic changes. However, in our previous paper, we already provided ultrastructural evidence of coupled mast cells and eosinophils in the tumor stroma of gastric carcinoma similar to that previously demonstrated in vitro through direct cell-cell adhesion [23]. Receptor/ligand couples between mast cells and eosinophils 

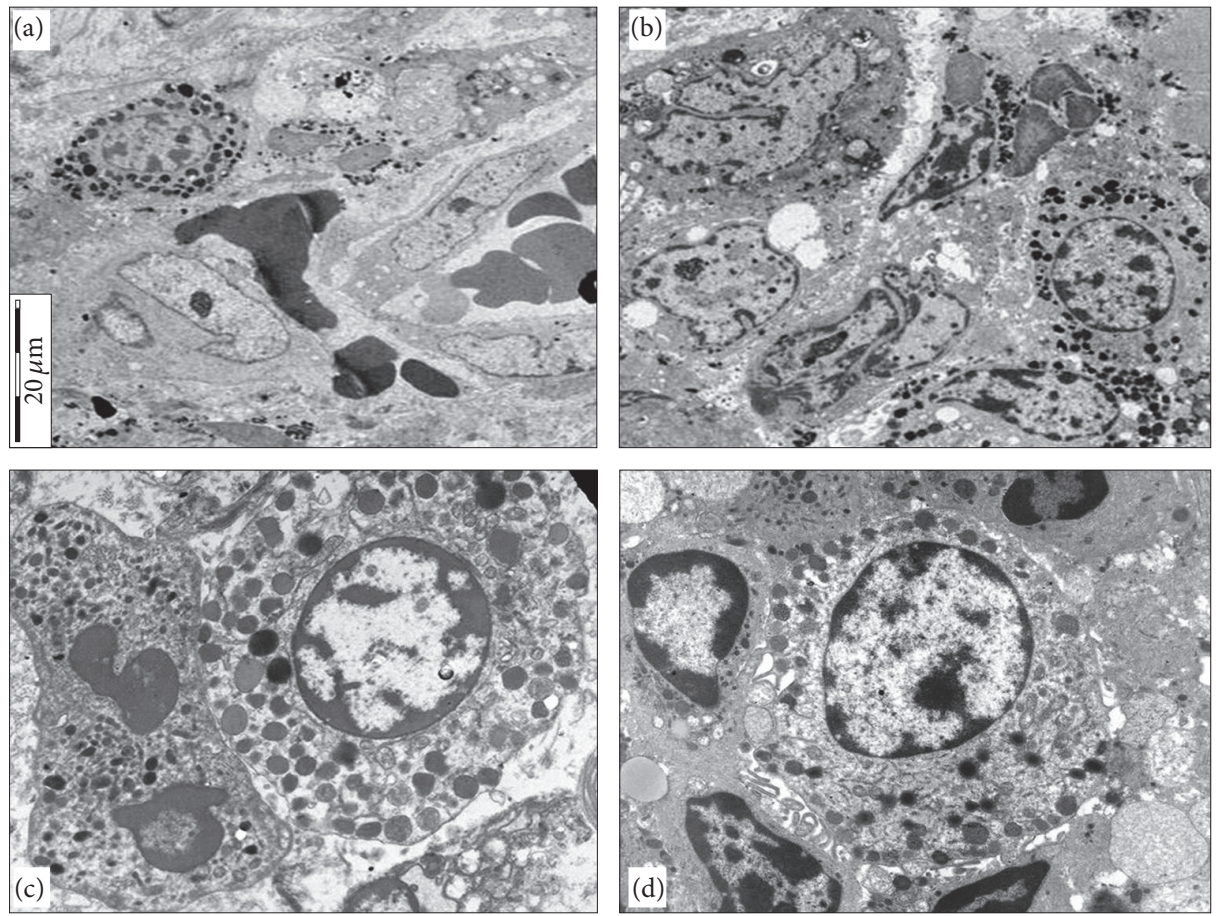

FIGURE 2: Mast cell showing a full complement of granules is in contact with a neutrophil, characterized by early apoptotic changes ((a), $\times 4000)$; another example of aggregation between mast cells and apoptotic neutrophils in the tumor stroma of a gastric adenocarcinoma ((b), $\times 4000$ ); mast cell established pointed or flat areas of membrane contact with a neutrophil showing early apoptotic changes. Mast cell exhibits cytoplasmic microtubules, some of which are in close vicinity to granules $((\mathrm{c}), \times 10000)$; neutrophil satellitosis around mast cell showing peripheral location of granules and cytoplasmic microtubules. Some neutrophils show early apoptotic changes $((d), \times 6000)$.

including CD48/2B4, CD226/CD112, and LFA-1/ICAM-1 were also previously described [24].

Receptors-ligand pair that may confirm the cross-talk between mast cells and neutrophils has not been reported thus far. Ultrastructurally, the analysis of degranulation in mast cells revealed two basic characteristic morphologic patterns, identified as massive anaphylactic degranulation and piecemeal degranulation [25]. The first one, the anaphylactic degranulation, is characterized by rapid secretion (within a few minutes) via degranulation channels or by direct fusion of granule containers with the cell membrane [25]. The second one, the piecemeal degranulation, presents partially or completely empty granule chambers in the absence of intergranular fusion [25]. Moreover, together with anaphylactic or piecemeal degranulation, granular contents of mast cells can be released in small portions by an alternative mechanism, called kiss-and-run fusion, involving transient opening of a fusion pore for the release of some or all of the granules without loss of mast cell integrity [26]. Therefore, although inconclusive, our ultrastructural findings in GC allow excluding mast cells anaphylactic degranulation and/or piecemeal degranulation and hypothesizing "kiss-and-run" mechanism. In fact, no evidence of massive anaphylactic type degranulation was found, while only a focal loss of granular density without fusion of intergranular membranes (piecemeal type) was encountered. Moreover, most of mast cell granules were intact and appeared to concentrate peripherally, sometimes near to plasma membrane, suggesting transient opening of a fusion pore, such as that hypothesized in the kiss-and-run fusion mechanism.

To date, degranulation of mast cells has not been linked to apoptosis of neutrophils, although mast cells were reported to induce apoptosis in vascular smooth muscle cells, endothelial cells, cardiomyocytes, and macrophages in vitro [27-30]. Both tryptase and chymase were suggested not only to be proapoptotic by themselves but also to potentiate the proapoptotic action of histamine [27]. Therefore, we can hypothesize that mast cells can firstly enhance and later suppress neutrophil activity in GC stroma. Accordingly, mast cells are responsible for the increased neutrophil infiltration during the inflammatory phase in other human pathological conditions, such as wound repair mechanism [31,32]. Therefore, cross-talk between mast cell and neutrophils, observed in the neoplastic stroma of GC, exhibits interesting analogy with the inflammatory phase of wound healing.

\section{Conclusion}

In the present study, a close morphological association between mast cells and neutrophils was ultrastructurally documented in a series of GC. The analysis of degranulation in mast cells suggested that their granular content can be released in small portions by a mechanism called kissand-run fusion, which is alternative to well-known massive anaphylactic or piecemeal degranulation. The physiopathological relationship between mast cells and neutrophils in 
GC requires further investigation and its potential prognostic significance needs to be clarified.

\section{Disclosure}

The authors alone are responsible for the content and writing of the paper.

\section{Competing Interests}

The authors report no competing interests.

\section{References}

[1] A. Ieni, V. Barresi, G. Giuffrè et al., "HER2 status in advanced gastric carcinoma: a retrospective multicentric analysis from Sicily," Oncology Letters, vol. 6, no. 6, pp. 1591-1594, 2013.

[2] A. Ieni, V. Barresi, L. Rigoli, R. A. Caruso, and G. Tuccari, "HER2 status in premalignant, early, and advanced neoplastic lesions of the stomach," Disease Markers, vol. 2015, Article ID 234851, 10 pages, 2015.

[3] V. Barresi, G. Branca, A. Ieni, L. Rigoli, G. Tuccari, and R. A. Caruso, "Phagocytosis (cannibalism) of apoptotic neutrophils by tumor cells in gastric micropapillary carcinomas," World Journal of Gastroenterology, vol. 21, no. 18, pp. 5548-5554, 2015.

[4] A. Ieni, V. Barresi, L. Rigoli, F. Fedele, G. Tuccari, and R. A. Caruso, "Morphological and cellular features of innate immune reaction in helicobacter pylori gastritis: a brief review," International Journal of Molecular Sciences, vol. 17, no. 1, article 109, 2016.

[5] K. Lee, H. Hwang, and K. T. Nam, "Immune response and the tumor microenvironment: how they communicate to regulate gastric cancer," Gut and Liver, vol. 8, no. 2, pp. 131-139, 2014.

[6] M. F. Dixon, R. M. Genta, J. H. Yardley, and P. Correa, “Classification and grading of gastritis. The updated Sydney System. International Workshop on the Histopathology of Gastritis, Houston 1994," The American Journal of Surgical Pathology, vol. 20, no. 10, pp. 1161-1181, 1996.

[7] S. Nakajima, N. Bamba, and T. Hattori, "Histological aspects and role of mast cells in Helicobacter pylori-infected gastritis," Alimentary Pharmacology and Therapeutics, vol. 20, supplement 1, pp. 165-170, 2004.

[8] V. Necchi, R. Manca, V. Ricci, and E. Solcia, "Evidence for transepithelial dendritic cells in human $\mathrm{H}$. pylori active gastritis," Helicobacter, vol. 14, no. 3, pp. 208-222, 2009.

[9] R. A. Caruso, A. Parisi, C. Crisafulli et al., "Intraepithelial infiltration by mast cells in human Helicobacter pylori active gastritis," Ultrastructural Pathology, vol. 35, no. 6, pp. 251-255, 2011.

[10] Cancer Genome Atlas Research Network, "Comprehensive molecular characterization of gastric adenocarcinoma," Nature, vol. 513, pp. 202-209, 2014.

[11] S. J. Galli, N. Borregaard, and T. A. Wynn, "Phenotypic and functional plasticity of cells of innate immunity: macrophages, mast cells and neutrophils," Nature Immunology, vol. 12, no. 11, pp. 1035-1044, 2011.

[12] S. J. Galli and M. Tsai, "Mast cells in allergy and infection: versatile effector and regulatory cells in innate and adaptive immunity, European Journal of Immunology, vol. 40, no. 7, pp. 1843$1851,2010$.
[13] Y. Wang and H. Thorlacius, "Mast cell-derived tumour necrosis factor- $\alpha$ mediates macrophage inflammatory protein-2induced recruitment of neutrophils in mice," British Journal of Pharmacology, vol. 145, no. 8, pp. 1062-1068, 2005.

[14] F. Doener, A. Michel, S. Reuter et al., "Mast cell-derived mediators promote murine neutrophil effector functions," International Immunology, vol. 25, no. 10, pp. 553-561, 2013.

[15] E. I. Egozi, A. M. Ferreira, A. L. Burns, R. L. Gamelli, and L. A. DiPietro, "Mast cells modulate the inflammatory but not the proliferative response in healing wounds," Wound Repair and Regeneration, vol. 11, no. 1, pp. 46-54, 2003.

[16] P. Martin and R. Nunan, "Cellular and molecular mechanisms of repair in acute and chronic wound healing," British Journal of Dermatology, vol. 173, no. 2, pp. 370-378, 2015.

[17] G. Gri, B. Frossi, F. D’Inca et al., "Mast cell: an emerging partner in immune interaction," Frontiers in Immunology, vol. 3, article 120, 2012.

[18] M. Elishmereni, N. Fyhrquist, R. Singh Gangwar, S. Lehtimäki, H. Alenius, and F. Levi-Schaffer, "Complex 2B4 regulation of mast cells and eosinophils in murine allergic inflammation," Journal of Investigative Dermatology, vol. 134, no. 12, pp. 29282937, 2014.

[19] R. S. Gangwar and F. Levi-Schaffer, "Eosinophils interaction with mast cells: the allergic effector unit," Methods in Molecular Biology, vol. 1178, pp. 231-249, 2014.

[20] M. Elishmereni, H. T. Alenius, P. Bradding et al., "Physical interactions between mast cells and eosinophils: a novel mechanism enhancing eosinophil survival in vitro," Allergy, vol. 66, no. 3, pp. 376-385, 2011.

[21] J.-Y. Jung, A. Yasoshima, J. Saegusa, H. Nakayama, and K. Doi, "Ultrastructural features of mast cells in picryl chloride (PCL)induced contact dermatitis in IQI/Jic mice," Experimental and Toxicologic Pathology, vol. 54, no. 4, pp. 265-271, 2003.

[22] P. Laurèn, "The two histological main types of gastric carcinoma: diffuse and so-called intestinal-type carcinoma. An attempt at a histoclinical classification," Acta Pathologica et Microbiologica Scandinavica, vol. 64, pp. 31-49, 1965.

[23] R. A. Caruso, F. Fedele, V. Zuccalà, M. G. Fracassi, and A. Venuti, "Mast cell and eosinophil interaction in gastric carcinomas: ultrastructural observations," Anticancer Research, vol. 27, no. 1, pp. 391-394, 2007.

[24] I. Bachelet, A. Munitz, D. Mankutad, and F. Levi-Schaffer, "Mast cell costimulation by CD226/CD112 (DNAM-1/Nectin2): a novel interface in the allergic process," The Journal of Biological Chemistry, vol. 281, no. 37, pp. 27190-27196, 2006.

[25] A. M. Dvorak, "Ultrastructural studies of human basophils and mast cells," Journal of Histochemistry and Cytochemistry, vol. 53, no. 9, pp. 1043-1070, 2005.

[26] U. Blank, I. K. Madera-Salcedo, L. Danelli et al., "Vesicular trafficking and signaling for cytokine and chemokine secretion in mast cells," Frontiers in Immunology, vol. 5, article 453, 2014.

[27] H. M. Heikkilä, S. Lätti, M. J. Leskinen, J. K. Hakala, P. T. Kovanen, and K. A. Lindstedt, "Activated mast cells induce endothelial cell apoptosis by a combined action of chymase and tumor necrosis factor- $\alpha$," Arteriosclerosis, Thrombosis, and Vascular Biology, vol. 28, no. 2, pp. 309-314, 2008.

[28] M. Leskinen, Y. Wang, D. Leszczynski, K. A. Lindstedt, and P. T. Kovanen, "Mast cell chymase induces apoptosis of vascular smooth muscle cells," Arteriosclerosis, Thrombosis, and Vascular Biology, vol. 21, no. 4, pp. 516-522, 2001. 
[29] M. Hara, A. Matsumori, K. Ono et al., "Mast cells cause apoptosis of cardiomyocytes and proliferation of other intramyocardial cells in vitro," Circulation, vol. 100, no. 13, pp. 1443-1449, 1999.

[30] I. Bot, S. C. A. de Jager, A. Zernecke et al., "Perivascular mast cells promote atherogenesis and induce plaque destabilization in apolipoprotein E-deficient mice," Circulation, vol. 115, no. 19, pp. 2516-2525, 2007.

[31] H. F. Dvorak, "Tumors: wounds that do not heal-redux," Cancer Immunology Research, vol. 3, no. 1, pp. 1-11, 2015.

[32] T. Marichal, M. Tsai, and S. J. Galli, "Mast cells: potential positive and negative roles in tumor biology," Cancer Immunology Research, vol. 1, no. 5, pp. 269-279, 2013. 


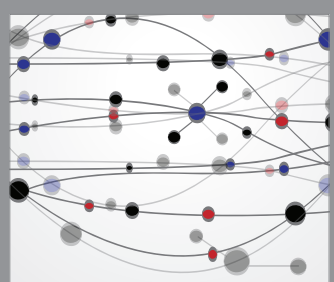

The Scientific World Journal
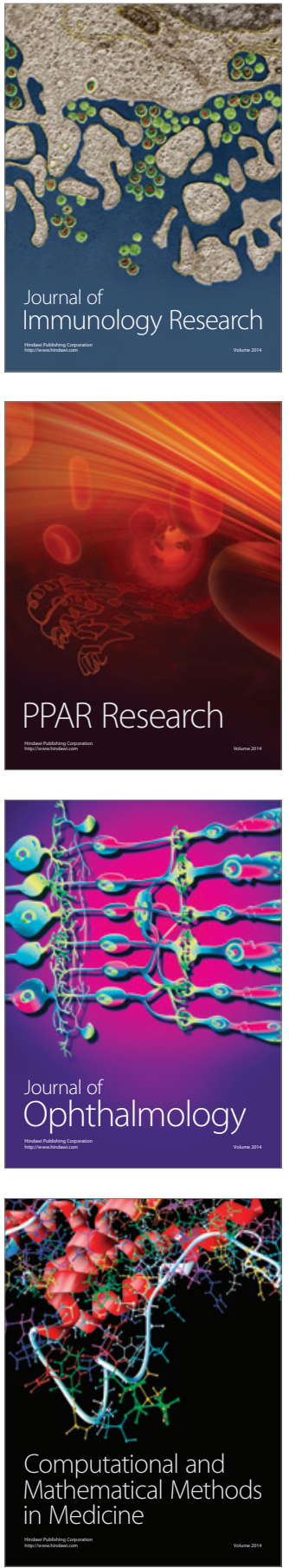

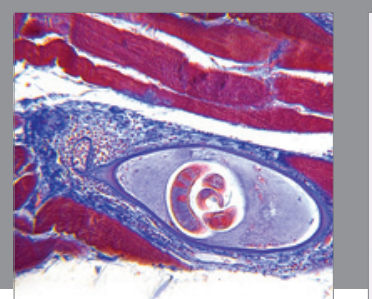

Gastroenterology Research and Practice

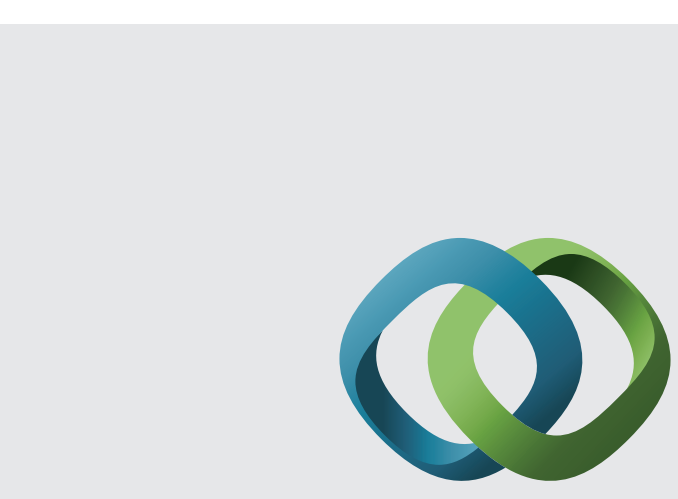

\section{Hindawi}

Submit your manuscripts at

http://www.hindawi.com
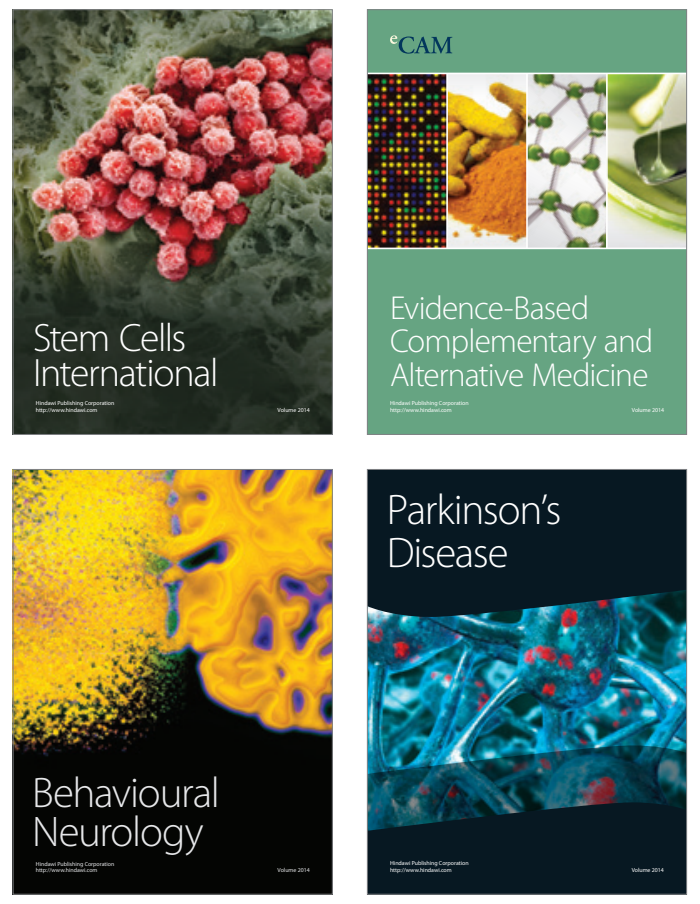
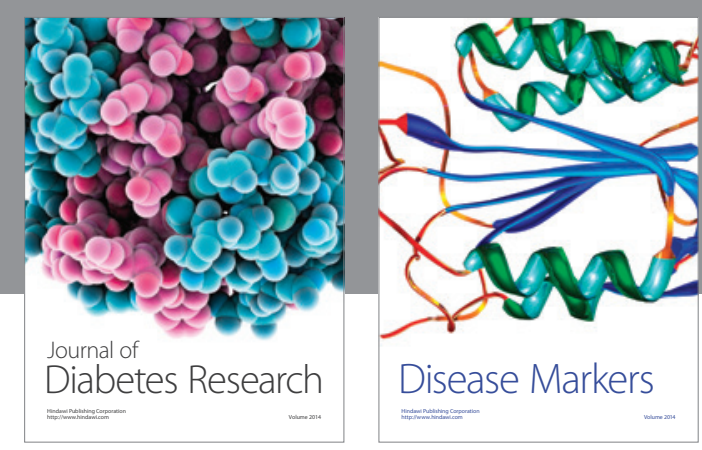

Disease Markers
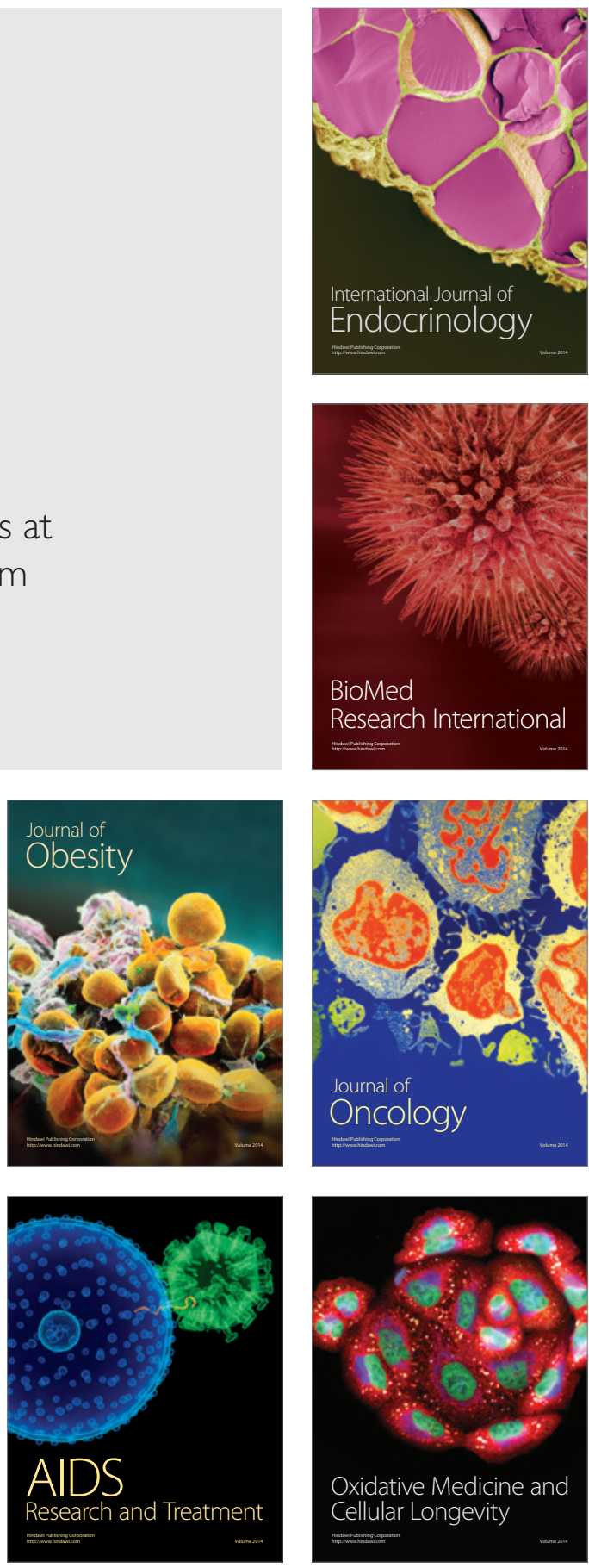